\title{
Formation and Rupture of the Internal Carotid Artery Aneurysm after Multiple Courses of Intensity-Modulated Radiation Therapy for Management of the Skull Base Ewing Sarcoma/ PNET: Case Report
}

\author{
Manabu Tamura ${ }^{1}$ Kasei Kogo ${ }^{2}$ Osamu Masuo ${ }^{3}$ Yoshinori Oura ${ }^{3}$ Hiroyuki Matsumoto ${ }^{3}$ Koji Fujita ${ }^{3}$ \\ Naoyuki Nakao ${ }^{3}$ Yuji Uematsu ${ }^{3}$ Toru Itakura $^{3}$ Mikhail Chernov ${ }^{1}$ Motohiro Hayashi ${ }^{1}$ \\ Yoshihiro Muragaki ${ }^{1}$ Hiroshi Iseki ${ }^{1}$
}

${ }^{1}$ Faculty of Advanced Techno-Surgery, Institute of Advanced Biomedical Engineering and Science, Tokyo Women's Medical University, Tokyo, Japan

${ }^{2}$ Kumamoto Radiosurgery Clinic, Kumamoto, Japan

${ }^{3}$ Department of Neurological Surgery, Wakayama Medical University, Wakayama, Japan

\begin{abstract}
Address for correspondence Manabu Tamura, MD, DMSc, Faculty of Advanced Techno-Surgery, Institute of Advanced Biomedical Engineering and Science, Tokyo Women's Medical University, 8-1 Kawada-cho, Shinjuku-ku, Tokyo 162-8666, Japan

(e-mail: Tamura.Manabu@twmu.ac.jp).
\end{abstract}

J Neurol Surg Rep 2013;74:111-117.

\begin{abstract}
Keywords

- malignant skull base tumor

- Ewing sarcoma/PNET

- intensity-modulated radiation therapy

- complication

- aneurysm formation

- radiation-induced aneurysm

Background Aneurysm formation after stereotactic irradiation of skull base tumors is rare. The formation and rupture of an internal carotid artery (ICA) aneurysm in a patient with skull base Ewing sarcoma/primitive neuroectodermal tumor (PNET), who underwent surgery followed by multiple courses of intensity-modulated radiation therapy (IMRT) and chemotherapy, is described.

Case Description A 25-year-old man presented with a sinonasal tumor with intraorbital and intracranial growth. At that time cerebral angiography did not reveal any vascular abnormalities. The lesion was resected subtotally. Histopathologic diagnosis was Ewing sarcoma/PNET. The patient underwent multiple courses of chemotherapy and three courses of IMRT at 3, 28, and 42 months after initial surgery. The total biologically effective dose delivered to the right ICA was 220.2 Gy. Seven months after the third IMRT, the patient experienced profound nasal bleeding that resulted in hypovolemic shock. Angiography revealed a ruptured right C4-C5 aneurysm and irregular stenotic changes of the ICA. Lifesaving endovascular trapping of the right ICA was done. The patient recovered well after surgery but died due to tumor recurrence 6 months later.

Conclusion Excessive irradiation of the ICA may occasionally result in aneurysm formation, which should be borne in mind during stereotactic irradiation of malignant skull base tumors.
\end{abstract}

received

December 13, 2012

accepted after revision

July 8, 2013

published online

October 22, 2013
DOI http://dx.doi.org/

10.1055/s-0033-1358379.

ISSN 2193-6358. (c) 2013 Georg Thieme Verlag KG

Stuttgart · New York
License terms

(ㄷ) (i) $\ominus$ (\$) 


\section{Introduction}

Fractionated radiotherapy (FRT) and stereotactic radiosurgery (SRS) are approved management options for skull base tumors, both benign and malignant. It may be applied as a definitive treatment or be a part of combined management of the neoplasm. Contemporary equipment provides an opportunity for very precise delivery of the efficient therapeutic doses to the target while preserving adjacent functionally important anatomical structures. Nevertheless, if cranial nerves, arteries, or veins are directly involved in the pathologic process, their inclusion into a high-dose irradiation area may be unavoidable. It may potentially lead to more or less pronounced treatment-associated morbidity. Severe arterial stenosis, aneurysm formation, and rupture were infrequently reported after conventional FRT or SRS of various intracranial lesions. ${ }^{1-5}$ In particular, formation and rupture of internal carotid artery (ICA) aneurysms were noted after irradiation of skull base tumors. ${ }^{6-10}$ We describe a similar complication that occurred in a patient with a skull base Ewing sarcoma/primitive neuroectodermal tumor (PNET), who underwent combined treatment including multiple courses of intensity-modulated radiation therapy (IMRT). This case was reported previously and was published elsewhere in Japanese. ${ }^{11}$

\section{Case Description}

A 25-year-old man presented with nasal bleeding, progressive visual loss in his right eye, and right-sided facial pain. Magnetic resonance imaging (MRI) demonstrated a tumor entirely filling the right ethmoid sinus with additional extension into the superior part of the maxillary sinus, orbit, and intracranial space ( - Fig. 1). At that time cerebral angiography did not show any vascular abnormalities ( - Fig. 2). Within the next few days, the patient's visual acuity deteriorated rapidly, and he eventually became blind in the right eye. Emergent surgical resection of the neoplasm was performed via the extended transbasal interhemispheric approach. Subtotal
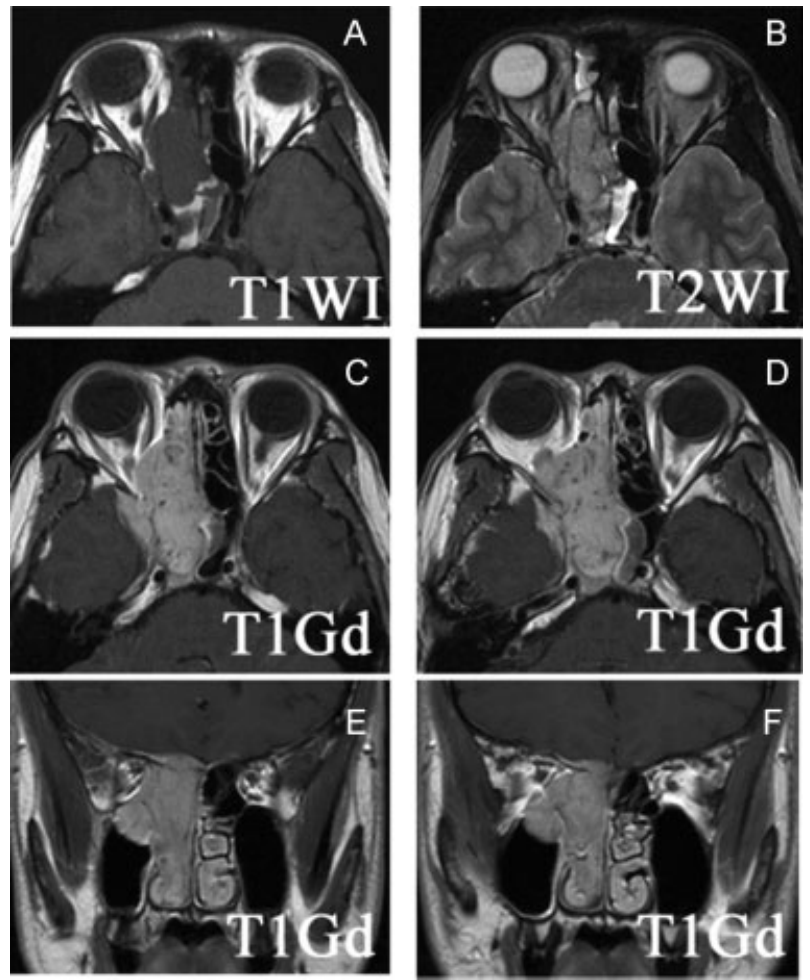

Fig. 1 (A) T1-weighted, (B) T2-weighted, (C, D) postcontrast axial, and $(\mathrm{E}, \mathrm{F})$ coronal T1-weighted magnetic resonance images demonstrate the extensive sinonasal tumor with intraorbital and intracranial growth.

removal of the lesion was attained, leaving its remnant within the right cavernous sinus. During surgery, the supraclinoid portion of the right ICA was completely freed from tumor encasement. The patient had an uneventful postoperative recovery and showed nearly complete restoration of the visual acuity and field of the right eye. Histopathologic examination of the pathologic tissue revealed that the tumor was composed of small round cells lying beneath the mucous membrane. The periodic acid-Schiff staining was positive, as
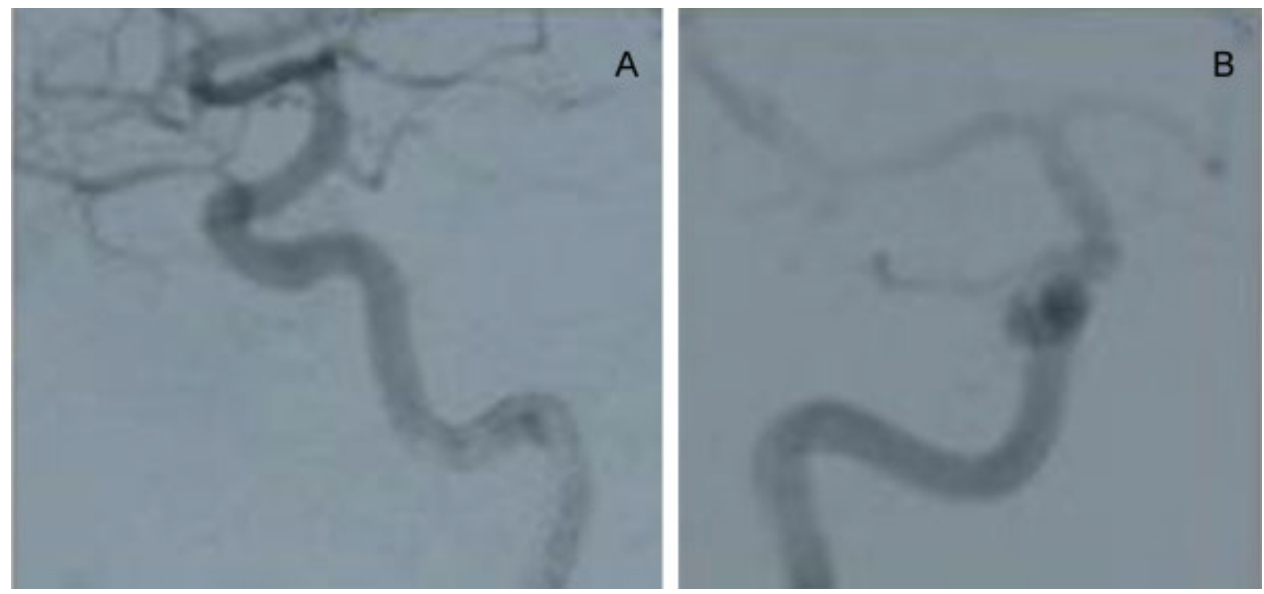

Fig. 2 (A) Lateral and (B) oblique projections of the right internal carotid artery angiogram at the time of diagnosis. No vascular abnormalities are evident. 

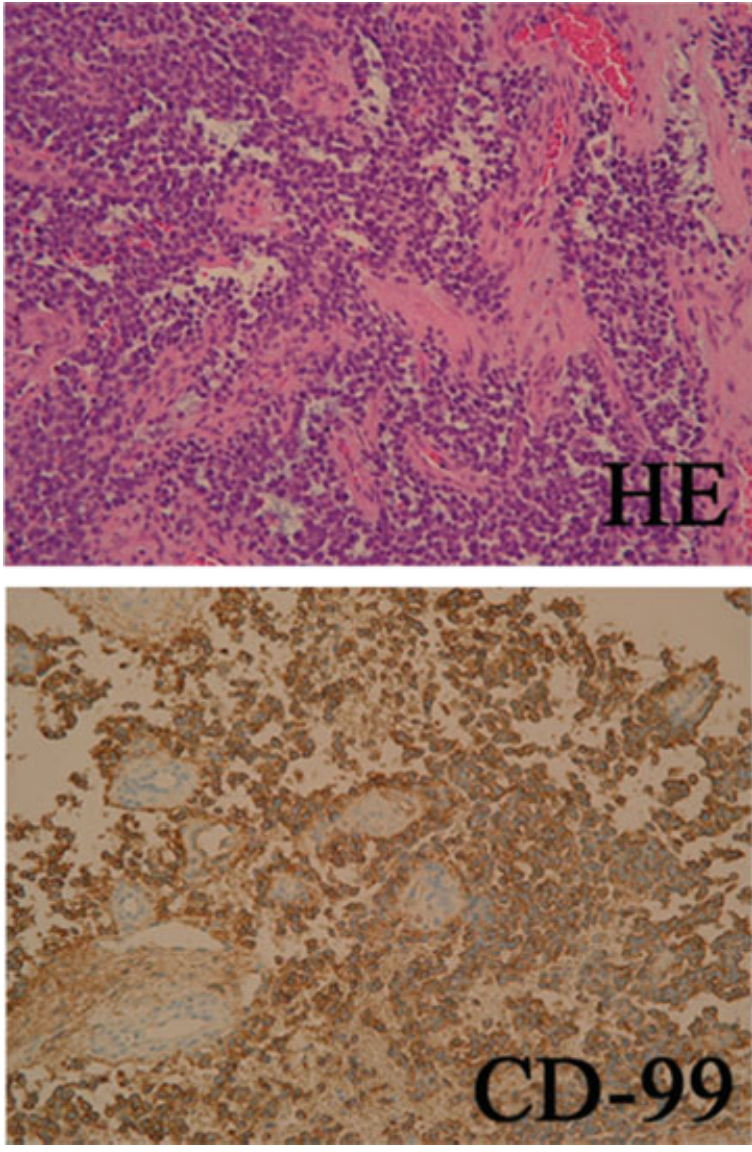

Fig. 3 Microphotographs of the histopathologic specimen stained with hematoxylin and eosin (HE) and CD-99: highly cellular tumor composed of small round cells with hyperchromatic nuclei and scanty cytoplasm diffusely showing perivascular arrangement.

well as immunohistochemical staining for vimentin and CD99 (-Fig. 3); neuronal and epithelial markers were negative. The MIB-1 index was assessed at $>20 \%$. A diagnosis of Ewing sarcoma/PNET was confirmed.

Postoperative whole-body 18F-fluorodeoxyglucose positron emission tomography (FDG-PET) showed no distant metastases. The patient underwent adjuvant multidrug chemotherapy that included three cycles of vincristine, doxorubicin, cyclophosphamide (VDC); two cycles of ifosfamide, etoposide (IE); and high-dose l-PAM (melphalan) and TEPA (thiotepa) combined with peripheral blood stem cell support. ${ }^{12-15}$ Radiation therapy was performed 3 months after surgery. To provide a high marginal dose to the tumor while avoiding excessive irradiation of the adjacent anatomical structures, particularly the optic nerve, multifractionated IMRT was applied. The treatment resulted in complete response of the residual intracranial tumor. No complications or side effects were noted. The general condition of the patient and his activities of daily life improved significantly. Visual functions were stable.

At 20 months after irradiation, the complete response of the neoplasm to treatment was maintained. Three months later, however, tumor recurrence in the superior part of the right maxillary sinus was revealed. The patient and his family declined the proposed surgical treatment. Therefore, seven additional cycles of chemotherapy, mainly using a modified ifosfamide, carboplatin, etoposide (ICE) regimen were performed. Because treatment did not result in tumor shrinkage, the second course of IMRT was done at 28 months after the initial surgery and resulted in a significant volumetric tumor response. Visual functions of the right eye were preserved.

Nevertheless, 11 months later, the patient started to complain of diplopia, limited extraocular movements, and pain in his right eye. A new recurrence was disclosed in the right superior orbital fissure in the vicinity of the previous irradiated field (-Fig. 4). Marked infiltration of the greater wing of the sphenoid bone was noted. Three cycles of chemotherapy based on VDC and IE were administered initially, followed by the third IMRT at 42 months after the initial surgery. At this time, preservation of visual function was considered impossible, and informed consent on this issue was obtained. Four additional cycles of chemotherapy were administered after irradiation. The treatment resulted in significant shrinkage of the orbital tumor and relief of the ocular pain, but it was accompanied by loss of vision in the right eye.

Development of prominent cytopenia required interruption of the chemotherapy. Additionally, the patient started to complain of severe headaches. T2-weighted MRI revealed an irregular area of hyperintense signal in the right temporal lobe that did not show radioisotope uptake on FDG-PET and was considered a radiation injury. Steroid therapy was initiated. Morphine and nonsteroidal anti-inflammatories were administered as required for pain control. At that time FDG-PET of the whole body showed no distant metastases.

Seven months after the third IMRT and a total of 49 months after initial manifestation of disease, the patient experienced profound nasal bleeding that resulted in hypovolemic shock and required intensive care. Computed tomography (CT) demonstrated massive intracerebral hemorrhage and pneumocephalus, and angiography revealed a ruptured right C4C5 carotid aneurysm, small ICA bifurcation aneurysm, and irregular stenotic changes of the vessel (-Fig. 5). Investigation was complicated by rerupture of the lesion. Lifesaving coiling of the right carotid artery was urgently done. The patient subsequently underwent treatment in the intensive care unit with artificial arterial hypertension and a blood transfusion. Within several days he nearly completely recovered. The pneumocephalus steadily resolved spontaneously.

One month after ICA occlusion and 7 months after the third IMRT, the follow-up MRI disclosed a new tumor recurrence on the right side of the sphenoid bone. It showed rapid progression and resulted in proptosis, facial deformity, and severe headache. The patient and his family were fully informed of the severity of the condition and the grim prognosis. They decided to avoid further treatment except for supportive care. The patient died 5 months later due to progressive growth of the neoplasm, in total 54 months after the initial manifestation of the disease. An autopsy was not performed. 

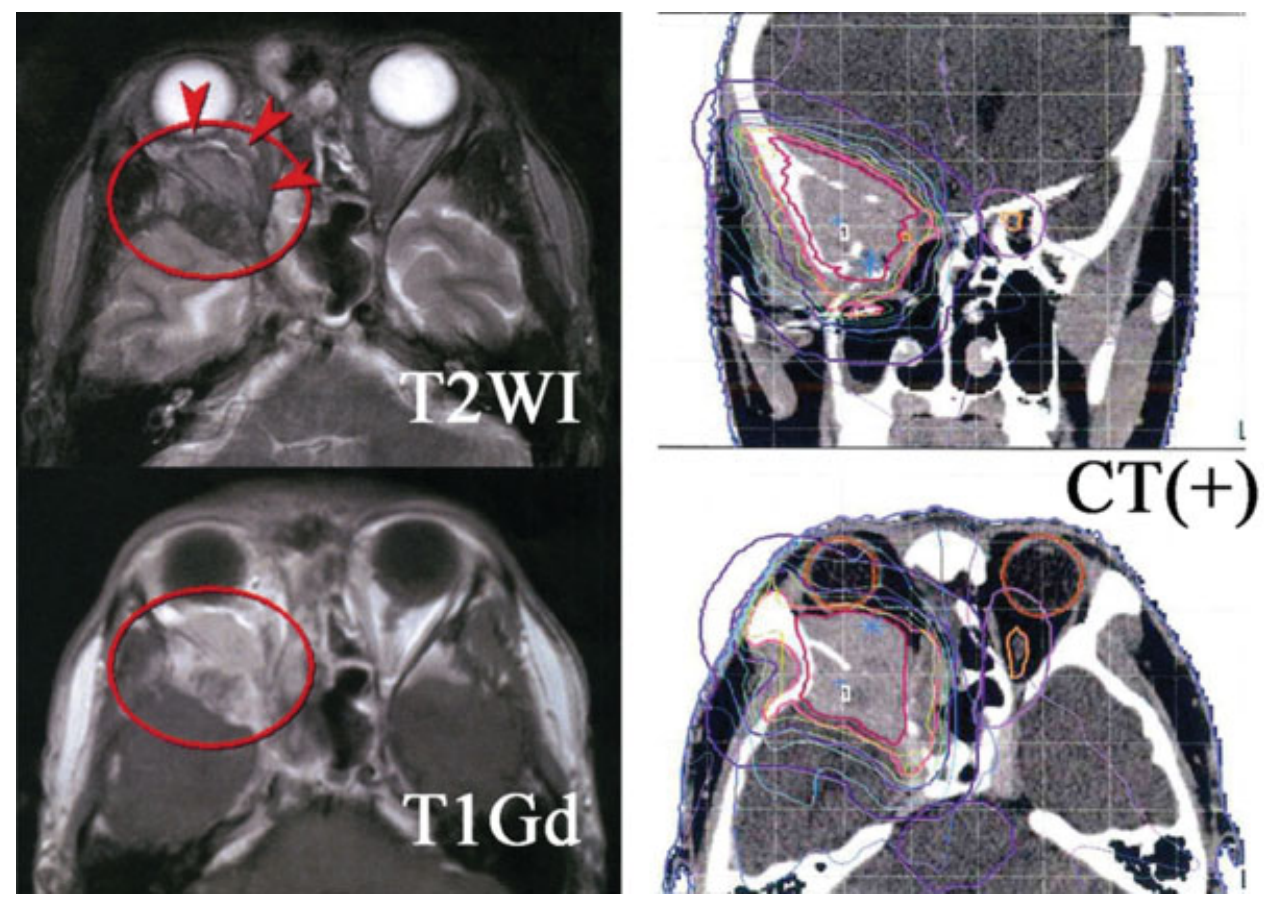

Fig. 4 Second recurrence of the tumor (red circles) with prominent intraorbital extension (arrowheads) 39 months after initial surgical resection (left) was managed with chemotherapy and the third intensity-modulated radiation therapy guided by contrast-enhanced computed tomography (right). The right internal carotid artery was included in the high-dose irradiation area.

\section{Details of Intensity-Modulated Radiation Therapy}

- Table 1 summarizes the details of the three courses of IMRT performed. The treatment was done using Novalis (Varian Medical Systems, Palo Alto, California, United States). Dose planning was based on contrast-enhanced CT images fused with MRI. The target volume was defined as a contrastenhanced region in the anterior skull base and paranasal sinuses corresponding to the tumor-occupied area and an additional 2-mm margin. The total biologically effective dose (BED) corresponding to conventional fractionated radiotherapy delivered during the three courses of IMRT to C4-C5 segments of the right ICA was $220.2 \mathrm{~Gy}$.

\section{Discussion}

Ewing sarcoma/PNET is highly malignant bone tumor that requires combined management including extensive sur- gical resection, chemotherapy, and irradiation. ${ }^{12-18}$ Its location on the skull base is extremely rare. ${ }^{19}$ Despite achievements of contemporary skull base surgery, gross total resection of such neoplasms is difficult to achieve and thus requires adjuvant therapy. In our patient, in addition to high-dose chemotherapy, the treatment included multiple courses of IMRT. It was done with the intention of avoiding radiation injury to the optic nerve located in close vicinity to the neoplasm and to provide high BED to maximize tumor control. Because the initial course of irradiation resulted in a complete volumetric tumor response, a similar treatment was also performed at the time of recurrence and was also successful. Such aggressive irradiation of the same area is definitely beyond current practice guidelines, but, particularly at the time of the second recurrence, it was administered as a last resort salvage treatment for the management of a highly aggressive tumor in a young
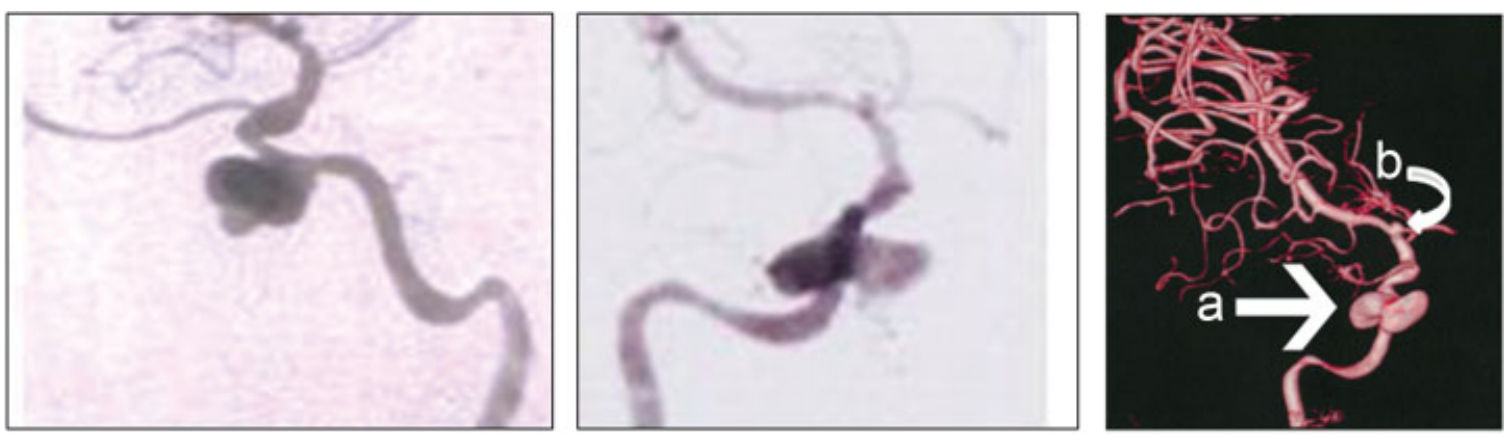

Fig. 5 Lateral (left), oblique (center), and three-dimensional reconstructed (right) digital subtraction angiography at the time of clinical deterioration accompanied by profound nasal bleeding demonstrated (a) ruptured right C4-C5 carotid aneurysm, and (b) small internal carotid artery bifurcation aneurysm and irregular stenotic changes of the vessel. 
Table 1 Multiple courses of intensity-modulated radiation therapy for management of the skull base Ewing sarcoma/primitive neuroectodermal tumor

\begin{tabular}{|c|c|c|c|}
\hline IMRT course ${ }^{a}$ & First & Second & Third \\
\hline Time from initial tumor resection (mo) & 3 & 28 & 42 \\
\hline Time to ICA aneurysm rupture (mo) & 46 & 24 & 7 \\
\hline Main target location & Right ethmoid sinus & $\begin{array}{l}\text { Superior part of the } \\
\text { right maxillary sinus }\end{array}$ & Right orbit \\
\hline Target size $(\mathrm{mm})$ & $75 \times 90 \times 75$ & $42 \times 72 \times 46$ & $57 \times 70 \times 57$ \\
\hline Target volume $\left(\mathrm{cm}^{3}\right)$ & 158.1 & 58.6 & 66.7 \\
\hline Central dose (Gy) & 54.9 & 62.3 & 60.0 \\
\hline Peripheral dose (Gy) & 45.0 & 53.5 & 52.8 \\
\hline Dose per fraction (Gy) & 3.0 & 3.6 & 3.5 \\
\hline $\mathrm{BED}^{\mathrm{b}}(\mathrm{SRS})$ & 19.7 & 22.0 & 22.0 \\
\hline $\mathrm{BED}^{\mathrm{b}}(\mathrm{FRT})$ & 54.7 & 61.0 & 60.0 \\
\hline $\begin{array}{l}\text { Dose to C4-C5 segments of the right ICA (Gy) } \\
\text { Total } \\
\text { Per fraction } \\
\text { BED }^{\mathrm{b}} \text { (FRT) }\end{array}$ & $\begin{array}{l}52.5 \\
3.5 \\
72.2\end{array}$ & $\begin{array}{l}55.5 \\
3.7 \\
79.1\end{array}$ & $\begin{array}{l}51.0 \\
3.4 \\
68.9\end{array}$ \\
\hline
\end{tabular}

Abbreviations: BED, biologically effective dose; FRT, fractionated radiation therapy (conventional); ICA, internal carotid artery; IMRT, intensitymodulated radiation therapy; SRS, stereotactic radiosurgery.

aEach course of IMRT consisted of 15 separate fractions.

${ }^{b}$ Calculations of the BEDs were made under the assumption of a tumor $\alpha: \beta$ ratio of 10 Gy and a tissue $\alpha: \beta$ ratio of 2 Gy.

patient. Overall, the first, second, and third courses of IMRT resulted in local progression-free survival of 23,11 , and 7 months, respectively. Of note, vision was preserved until the optic nerve was intentionally included in the high-dose irradiation area during management of the second local recurrence of the tumor. Nevertheless, the cumulative effect of multiple courses of IMRT led to several serious complications, among the most devastating was the formation and rupture of the ICA aneurysm, which was seemingly caused by excessive irradiation of the vessel wall.

There are several reports on aneurysm formation in the common carotid artery, as well as cervical and petrosal segments of ICA after radiotherapy of laryngeal carcinoma, nasopharyngeal carcinoma, and prolactinoma. ${ }^{6-10,20}$ It was found from 3 to 13 years after irradiation with a dose ranging from 60 to $72 \mathrm{~Gy}$, and it was sometimes associated with distant stenosis of the vessel or necrosis of the temporal bone. ${ }^{7,8,20}$ Multiple courses of high-dose FRT may be accompanied by a greater risk of such complications. ${ }^{6,9}$ Similarly, formation of intracranial aneurysms was reported on longterm follow-up after conventional FRT, stereotactic radiation therapy, or SRS of various brain tumors, as well as arteriovenous malformations. ${ }^{1,2,4,5}$ Although in most cases it occurred after wide-field irradiation with evident involvement of the affected vessel in the high-dose area, there was a report on ICA aneurysm formation 6 years after gamma knife radiosurgery of a vestibular schwannoma with a marginal dose of 12 $\mathrm{Gy}^{3}{ }^{3}$

It is impossible to define precisely the time of aneurysm formation in our patient. Moreover, although its main cause was seemingly high total BED delivered to the ICA, which during three courses of IMRT totaled $220.2 \mathrm{~Gy}$, possible injury of the vessel wall at the time of the initial surgical removal of the tumor or its direct effect by the malignant neoplasm could also have played an important role. There are multiple reports on iatrogenic intracranial traumatic aneurysms and pseudoaneurysms after microsurgical resection of skull base neoplasms and other neurosurgical procedures. ${ }^{21-25}$ Ciceri et al ${ }^{23}$ mentioned 52 such cases published from 1955 , most frequently affecting the ICA, usually after transsphenoidal surgery. It was speculated that "fusiform" lesions occur due to weakening of the adventitia during surgical peeling of the tumor from the artery, whereas "saccular" ones develop after more focal or complete laceration of the vessel. ${ }^{23}$ It was suggested that most traumatic intracranial aneurysms have symptomatic manifestations within 3 to 4 weeks after injury of the vessel wall. ${ }^{25}$ In our patient, however, the hemorrhagic presentation appeared 49 months after the initial tumor resection, which makes traumatic origin of the aneurysm less probable. Finally, intensive antitumor chemotherapy performed in our case could also have had some predisposing effects on the aneurysm formation, although such causative interrelationships are difficult to prove.

Patients with fusiform iatrogenic aneurysms seemingly may be followed without any intervention, and in many of them the neuroradiologic findings remain unchanged. ${ }^{23}$ Nevertheless, rupture of radiation-induced ICA aneurysm constitutes a life-threatening complication and requires emergency management. In such cases endovascular procedures may be considered the treatment of choice and usually provide favorable results. ${ }^{23,24}$ In our patient, rerupture 
during angiography required trapping of the vessel. Insufficient collateral blood flow may stipulate additional bypass surgery. ${ }^{6,10}$ Other options include the use of covered stent and coiling of the aneurysm. ${ }^{7,23,24}$

\section{Conclusion}

Use of IMRT for combined management of skull base Ewing sarcoma/PNET may result in a prominent volumetric response and may be effectively applied for control of the residual neoplasm after surgery, as well as at the time of local treatment failure. Nevertheless, the long-term prognosis in such cases is poor due to the malignant nature of the tumor and its propensity for local recurrence. Involvement of the cerebral arteries, particularly the ICA, into the neoplastic process may lead to their unavoidable inclusion into the high-dose irradiation area, which may occasionally result in aneurysm formation. It should be borne in mind during management of skull base neoplasms and the follow-up of patients after treatment. Investigation with magnetic resonance angiography or computed tomographic angiography may be reasonable in some cases.

\section{Acknowledgments}

The authors thank Dr. Shinji Kounami (Department of Pediatrics, Wakayama Medical University) for his contributions to the treatment of our patient. This work was partially supported by the Multidisciplinary Education and Research Center for Regenerative Medicine (MERCREM) under the Global COE Program of the Japan Ministry of Education, Culture, Sports, Science and Technology (MEXT).

\section{Note}

This case report was presented during the 19th Annual Meeting of the Japanese Society for Stereotactic Radiosurgery (July 30, 2010; Otsu, Japan) and 10th Biennial Congress of the International Stereotactic Radiosurgery Society (May 8-12, 2011; Paris, France).

\section{References}

1 Benson PJ, Sung JH. Cerebral aneurysms following radiotherapy for medulloblastoma. J Neurosurg 1989;70(4): 545-550

2 Yucesoy K, Feiz-Erfan I, Spetzler RF, Han PP, Coons S. Anterior communicating artery aneurysm following radiation therapy for optic glioma: report of a case and review of the literature. Skull Base 2004;14(3):169-173

3 Takao T, Fukuda M, Kawaguchi T, et al. Ruptured intracranial aneurysm following gamma knife surgery for acoustic neuroma. Acta Neurochir (Wien) 2006;148(12):1317-1318; discussion 1318

4 Yamaguchi S, Kato T, Takeda M, Ikeda H, Kitamura K. Ruptured distal anterior inferior cerebellar artery aneurysm following stereotactic irradiation for vestibular schwannoma: case report. Neurol Med Chir (Tokyo) 2009;49(5):202-205
5 Gross BA, Ropper AE, Du R. Vascular complications of stereotactic radiosurgery for arteriovenous malformations. Clin Neurol Neurosurg 2013;115(6):713-717

6 Cheng KM, Chan CM, Cheung YL, Chiu HM, Tang KW, Law CK. Endovascular treatment of radiation-induced petrous internal carotid artery aneurysm presenting with acute haemorrhage. A report of two cases. Acta Neurochir (Wien) 2001;143(4):351-355; discussion 355-356

7 Auyeung KM, Lui WM, Chow LC, Chan FL. Massive epistaxis related to petrous carotid artery pseudoaneurysm after radiation therapy: emergency treatment with covered stent in two cases. AJNR Am J Neuroradiol 2003;24(7):1449-1452

8 Lau WY, Chow CK. Radiation-induced petrous internal carotid artery aneurysm. Ann Otol Rhinol Laryngol 2005;114(12): 939-940

9 Cheng KY, Lee KW, Chiang FY, Ho KY, Kuo WR. Rupture of radiation-induced internal carotid artery pseudoaneurysm in a patient with nasopharyngeal carcinoma-spontaneous occlusion of carotid artery due to long-term embolizing performance. Head Neck 2008;30(8):1132-1135

10 Endo H, Fujimura M, Inoue T, et al. Simultaneous occurrence of subarachnoid hemorrhage and epistaxis due to ruptured petrous internal carotid artery aneurysm: association with transsphenoidal surgery and radiation therapy: case report. Neurol Med Chir (Tokyo) 2011;51(3):226-229

11 Tamura M, Kogo K, Masuo O, et al. The ruptured carotid artery pseudoaneurysm presenting as intractable epistaxis after repeated SRT for skull base Ewing PNET: case report. [in Japanese]. Stereotactic Radiotherapy 2011;15:35-44

12 Kushner BH, Meyers PA, Gerald WL, et al. Very-high-dose shortterm chemotherapy for poor-risk peripheral primitive neuroectodermal tumors, including Ewing's sarcoma, in children and young adults. J Clin Oncol 1995;13(11):2796-2804

13 Grier HE, Krailo MD, Tarbell NJ, et al. Addition of ifosfamide and etoposide to standard chemotherapy for Ewing's sarcoma and primitive neuroectodermal tumor of bone. N Engl J Med 2003; 348(8):694-701

14 Hotte SJ, Smith AM, Bramwell VH, Howson-Jan K. High-dose chemotherapy followed by peripheral and/or bone marrow stem cell transplant in patients with advanced sarcoma: experience of a Canadian centre. Sarcoma 2004;8(2-3):63-69

15 Miser JS, Krailo MD, Tarbell NJ, et al. Treatment of metastatic Ewing's sarcoma or primitive neuroectodermal tumor of bone: evaluation of combination ifosfamide and etoposide-a Children's Cancer Group and Pediatric Oncology Group study. J Clin Oncol 2004;22(14):2873-2876

16 Wilkins RM, Pritchard DJ, Burgert EO Jr, Unni KK. Ewing's sarcoma of bone. Experience with 140 patients. Cancer 1986;58(11): 2551-2555

17 Elomaa I, Blomqvist CP, Saeter G, et al. Five-year results in Ewing's sarcoma. The Scandinavian Sarcoma Group experience with the SSG IX protocol. Eur J Cancer 2000;36(7):875-880

18 van Doorninck JA, Ji L, Schaub B, et al. Current treatment protocols have eliminated the prognostic advantage of type 1 fusions in Ewing sarcoma: a report from the Children's Oncology Group. J Clin Oncol 2010;28(12):1989-1994

19 Louis DN, Ohgaki H, Wiestler OD, et al. The 2007 WHO classification of tumours of the central nervous system. Acta Neuropathol 2007;114(2):97-109

20 Koenigsberg RA, Grandinetti LM, Freeman LP, McCormick D, Tsai F. Endovascular repair of radiation-induced bilateral common carotid artery stenosis and pseudoaneurysms: a case report. Surg Neurol 2001;55(6):347-352

21 Dario A, Dorizzi A, Scamoni C, Cerati M, Balcone Grimaldi G. Iatrogenic intracranial aneurysm. Case report and review of the literature. J Neurosurg Sci 1997;41(2):195-202 
22 Grigorian A, Rajaraman V, Hunt CD. Traumatic intracranial aneurysms complicating anterior skull base surgery. J Craniomaxillofac Trauma 1998;4(4):10-14

23 Ciceri EFM, Regna-Gladin C, Erbetta A, et al. Iatrogenic intracranial pseudoaneurysms: neuroradiological and therapeutical considerations, including endovascular options. Neurol Sci 2006;27(5): $317-322$
24 Mathews SS, Kumar RM, Rupa V. Iatrogenic pseudoaneurysm: a rare complication of sinonasal surgery. Am J Otolaryngol 2011; 32(6):607-610

25 Chen Z, Zhang J, Miao H, Niu Y, Feng H, Zhu G. Delayed rupture of iatrogenic cerebral pseudoaneurysms after neurosurgical procedures: report of two cases. Clin Neurol Neurosurg 2013;115(8): 1552-1554 